\title{
Are short $\gamma$-ray bursts collimated? GRB 050709, a flare but no break
}

\author{
D. Watson, J. Hjorth, P. Jakobsson, D. Xu, J. P. U. Fynbo, J. Sollerman, C. C. Thöne, and K. Pedersen
}

\begin{abstract}
Dark Cosmology Centre, Niels Bohr Institute, University of Copenhagen, Juliane Maries Vej 30, 2100 Copenhagen $\varnothing$, Denmark e-mail: [darach; jens; pallja; dong; j fynbo; jesper; cthoene; kp] @astro.ku.dk
\end{abstract}

Received 6 April 2006 / Accepted 2 June 2006

\section{ABSTRACT}

\begin{abstract}
Context. From the small sample of afterglow lightcurves of short duration $\gamma$-ray bursts (GRBs), the decays are rapid, roughly following a power-law in time. It has been assumed that the afterglow emission in short GRBs is collimated in jets in the same way as in long GRBs.

Aims. An achromatic break in a short GRB afterglow lightcurve would therefore be strong evidence in favour of collimation in short GRBs.

Methods. We examine the optical lightcurve of the afterglow of the short GRB 050709, the only short GRB where a jet break has been claimed from optical data.

Results. We show that (1) the decay follows a single power-law from 1.4 to 19 days after the burst and has a decay index $\alpha=1.73_{-0.04}^{+0.11}$; (2) that an optical flare at $\sim 10$ days is required by the data, roughly contemporaneous with a flare in the X-ray data; and (3) that there is no evidence for a break in the lightcurve.

Conclusions. This means that so far there is no direct evidence for collimation in the outflows of short GRBs. The available limits on the collimation angles in short GRBs now strongly suggest much wider opening angles than found in long GRBs.
\end{abstract}

Key words. gamma rays: bursts - stars: neutron

\section{Introduction}

Great progress has been made in the past year on the origins of short-duration ( $<2$ s, Norris et al. 1984; Dezalay et al. 1992; Kouveliotou et al. 1993) $\gamma$-ray bursts (SGRBs), mostly due to the detection of the first afterglows of SGRBs at X-ray (Gehrels et al. 2005), optical (Hjorth et al. 2005b) and radio (Berger et al. 2005) wavelengths. Their detection in galaxies with little starformation (Fox et al. 2005; Berger et al. 2005; Prochaska et al. 2006; Gorosabel et al. 2006), and lack of an associated supernova (Hjorth et al. 2005a; Castro-Tirado et al. 2005), is in direct contrast to long-duration GRBs (LGRBs) which are associated with the deaths of massive stars (Galama et al. 1998; Stanek et al. 2003; Hjorth et al. 2003; Malesani et al. 2004). In fact, the recent SN-LGRB, SN 2006aj/GRB 060218 has resulted in a large body of new data on these objects (Pian et al. 2006; Campana et al. 2006; Sollerman et al. 2006; Modjaz et al. 2006; Cobb et al. 2006; Mirabal et al. 2006). In the past few months considerable data has been garnered on the afterglow properties of SGRBs. With these recent results, the range of distances to, and isotropic equivalent energies of, SGRBs has expanded (Levan et al. 2006; Soderberg et al. 2006).

It is generally assumed that SGRB afterglows have properties similar to the afterglows of LGRBs (e.g. Panaitescu et al. 2001; Lazzati et al. 2001; Fox et al. 2005; Panaitescu 2006; Covino et al. 2006; Berger et al. 2005). The afterglows of SGRBs do show fast, approximately power-law decays in X-ray and optical wavelengths, but with many strong deviations from a simple power-law model. These deviations are interpreted as energy injection or short-term flaring (Levan et al. 2006; La Parola et al. 2006; Soderberg et al. 2006). Much has been inferred about the collimation properties of SGRBs from the variations from a power-law in a single band (Fox et al. 2005; Berger et al. 2005; Soderberg et al. 2006). Given the strong flaring activity now known to exist in SGRB decays, it is reasonable to be cautious about such inferences. Indeed, in only one case to date has a positive claim been made for a jet break in an SGRB optical lightcurve, GRB 050709 (Fox et al. 2005).

In this paper we analyse the available data on the spectral and temporal properties of the afterglow of GRB 050709, the first SGRB where an optical afterglow was detected and where a claim for a jet break has been made. We then examine the limits on jet breaks in other SGRBs and compare the opening angles of SGRBs with LGRBs.

\section{The optical lightcurve of GRB 050709}

The optical-near-infrared lightcurve of the afterglow in any one band is sparsely sampled. But detections have been made in the $V, R, F 814 W$, and $K^{\prime}$ bands (Table 1 ), so we can create a reasonably sampled lightcurve over a long timescale with a little knowledge of the broadband spectrum. The $R$ and $F 814 W$ bands are the best-constrained data and drive a power-law fit to the data (Fig. 1). Fortunately, these bands are spectrally close, so that the colour-correction is small.

In long duration GRBs the afterglow continua are predominantly power-laws (e.g. Sari et al. 1998; Jakobsson et al. 2004; Willingale et al. 2004). It seems reasonable that the optical/NIR spectrum of GRB 050709 can be represented by a power-law shape especially over the small spectral range that dominates the lightcurve fit ( $R$ to $F 814 W$ ). Using the near-simultaneous $V$ 
Table 1. Optical observations of GRB 050709 in 2005. Colourcorrected fluxes used in Fig. 1 are given in Col. 5.

\begin{tabular}{rccccc}
\hline \hline $\begin{array}{r}\text { Observation } \\
\text { Date Time }\end{array}$ & $\begin{array}{c}\Delta t \\
\text { Days })\end{array}$ & Magnitude & Band & $\begin{array}{c}R \text {-band flux } \\
(\mu \mathrm{Jy})\end{array}$ \\
\hline \multicolumn{2}{c}{ July } \\
${ }^{a} 11$ & $08: 37$ & 1.4166 & $22.71 \pm 0.06$ & $R$ & $2.7 \pm 0.1$ \\
${ }^{a} 12$ & $07: 53$ & 2.3862 & $23.46 \pm 0.28$ & $R$ & $1.4_{-0.3}^{+0.4}$ \\
${ }^{b} 12$ & $09: 32$ & 2.4551 & $>23.25$ & $I$ & $<1.2$ \\
${ }^{b} 12$ & $09: 44$ & 2.4635 & $24.38 \pm 0.10$ & $V$ & $0.93_{-0.07}^{+0.08}$ \\
${ }^{b} 12$ & $09: 57$ & 2.4725 & $23.83 \pm 0.07$ & $R$ & $1.01 \pm 0.06$ \\
${ }^{b} 14$ & $07: 21$ & 4.3642 & $>25.00$ & $V$ & $<0.6$ \\
${ }^{b} 14$ & $07: 21$ & 4.3718 & $>24.10$ & $I$ & $<0.6$ \\
${ }^{c} 15$ & $13: 49$ & 5.6336 & $25.08 \pm 0.02$ & $F 814 W$ & $0.248 \pm 0.005$ \\
${ }^{c} 15$ & $14: 06$ & 5.6454 & $22.1 \pm 0.7$ & $K^{\prime}$ & $0.2_{-0.1}^{+0.2}$ \\
${ }^{a} 17$ & $07: 46$ & 7.3812 & $>24.1$ & $R$ & $<0.8$ \\
${ }^{c} 19$ & $17: 11$ & 9.7739 & $25.84 \pm 0.05$ & $F 814 W$ & $0.123 \pm 0.006$ \\
${ }^{a} 27$ & $09: 07$ & 17.4378 & $>24.0$ & $R$ & $<0.9$ \\
${ }^{c} 28$ & $13: 48$ & 18.6329 & $27.81 \pm 0.27$ & $F 814 W$ & $0.020_{-0.004}^{+0.006}$ \\
${ }^{a} 29$ & $09: 30$ & 19.4536 & $>23.8$ & $R$ & $<1.1$ \\
${ }^{a} 30$ & $02: 37$ & 20.1669 & $>25.20$ & $V$ & $<0.5$ \\
${ }^{b} 30$ & $02: 54$ & 20.1787 & $>25.00$ & $R$ & $<0.4$ \\
${ }^{b} 30$ & $04: 10$ & 20.2315 & $>23.50$ & $I$ & $<1.0$ \\
August & & & & \\
${ }^{c} 13$ & $15: 17$ & 34.6947 & $>28.1$ & $F 814 W$ & $<0.015$ \\
\hline
\end{tabular}

${ }^{a}$ Danish $1.54 \mathrm{~m}$ (Hjorth et al. 2005b); ${ }^{b}$ VLT (Covino et al. 2006); ${ }^{c}$ HST and Subaru (Fox et al. 2005).

and $R$ (2.4 days), and $F 814 W$ and $K^{\prime}$ (5.6 days) observations, the spectral index of the power-law $\left(F_{v} \propto v^{-\beta}\right)$ was $\beta_{\mathrm{O}}=1.7 \pm 0.8$, and $\beta_{\mathrm{O}}=1.2 \pm 0.7$, respectively. Combining these data gives $\beta_{\mathrm{O}}=1.4 \pm 0.5$. The upper limit in the $I$ band at 2.4 days is consistent with this spectral index. This is bluer than the $\beta_{\mathrm{O}}=2.3 \pm 0.7$ derived by Covino et al. (2006), but still within the $1 \sigma$ error bounds. All detections before 5 days in the literature have assumed a zero flux from the afterglow at about a week. To correct for this, a small flux derived from the late afterglow (using the HST lightcurve) was added to the early flux values. The offset added to the early data is partly responsible for the bluer spectral index derived here.

Using this power-law spectrum with $\beta_{\mathrm{O}}=1.4$, the data were converted to fluxes at the effective wavelength of the $R$ band. The precise value of the colour correction does not substantially affect the lightcurve; values of $\beta_{\mathrm{O}}$ between 1.0 and 2.4 give very similar results. This relative insensitivity to the colour correction is because $\beta_{\mathrm{O}}$ is derived from the same wavelength range as the lightcurve data and, as mentioned above, because the lightcurve fit is driven primarily by the $R$-band and $F 814 W$ data, where the wavelength separation is quite small.

The resulting lightcurve was then fit with a single power-law (Fig. 1), yielding a poor fit regardless of the colour correction $\left(\chi^{2}=27.6\right.$ for 6 degrees of freedom). The poor fit was entirely due to the second HST datapoint at 9.8 days. A broken powerlaw improved the fit slightly, but still the fit was unacceptable $\left(\chi^{2}=16.2\right.$ for 4 degrees of freedom $)$ and in fact required a flattening rather than a steepening of the decay. However, excluding the second HST detection from the dataset allowed a good fit to be obtained with a single power-law $\left(F(t) \propto t^{-\alpha}\right)$ with a moderately steep decay index $\alpha_{\mathrm{O}}=1.73 \pm 0.04\left(\chi^{2}=6.2\right.$ for 5 degrees of freedom). Adding the uncertainty from the colour correction gives $\alpha_{\mathrm{O}}=1.73_{-0.04}^{+0.11}$

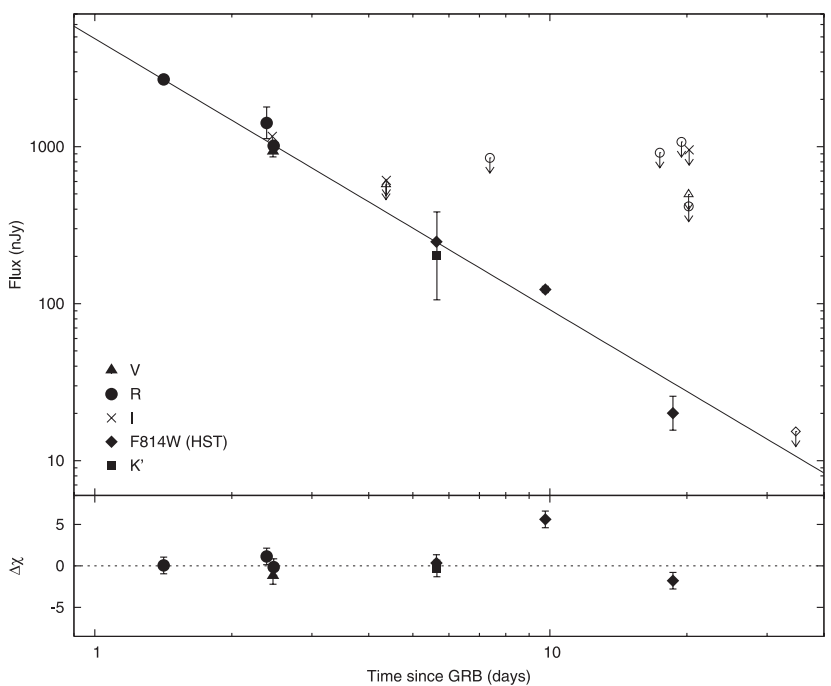

Fig. 1. The optical lightcurve of the short GRB 050709. A single powerlaw decay $\left(\alpha_{\mathrm{O}}=1.73\right)$ has been fit to the data excluding the second HST detection at 9.8 days. An acceptable fit is obtained only when this datapoint is excluded. Data from different bands have been corrected to the $R$-band flux using the best-fit power-law spectrum. The fit subtracted from the data (residuals) in units of $\Delta \chi$ are plotted in the lower panel.

\subsection{Comparison with $X$-rays}

The first Chandra observation shows a clear detection of the source with $\sim 50$ counts (Fox et al. 2005). Assuming a powerlaw model with Galactic absorption, the power-law spectral index is $\beta_{\mathrm{X}}=1.6 \pm 0.3$, consistent with $\beta_{\mathrm{O}}$ derived above. The $0.3-8.0 \mathrm{keV}$ flux is $7 \pm 2 \times 10^{-15} \mathrm{erg} \mathrm{cm}^{-2} \mathrm{~s}^{-1}(2 \pm 1 \times$ $10^{-16} \mathrm{erg} \mathrm{cm}^{-2} \mathrm{~s}^{-1} \mathrm{keV}^{-1}$ or $0.08_{-0.04}^{+0.06} \mathrm{nJy}$ at $5 \mathrm{keV}$ ). The opticalto-X-ray spectral index is then $\beta_{\mathrm{OX}}=1.2 \pm 0.1$ at 2.45 days. This spectral index is consistent with all of the spectral indices derived in the optical/NIR. The data are therefore consistent with a power-law spectrum with a single power-law index from the NIR to the X-ray regime.

\section{Implications for short GRBs}

It has already been noted that the HST data are not consistent with a single power-law decay in GRB 050709 and it was suggested that the second detection with HST represented a break to a steeper decay rate, consistent with a jet break (Fox et al. 2005). It is clear from this analysis of all the available data, that there is no evidence for a break in the lightcurve of GRB 050709. The HST datapoint at 9.8 days, instead, represents a flare or a rebrightening in the optical. This is not surprising empirically, in light of the probable flare in the X-ray data for this burst (Fox et al. 2005) at 16 days, as well as the rebrightenings observed in other SGRBs: GRB 050724 (Barthelmy et al. 2005; Berger et al. 2005), GRB 051210 (La Parola et al. 2006), GRB 051221A (Soderberg et al. 2006), GRB 060121 Levan et al. (2006). The $\mathrm{X}$-ray flare may be directly related to the optical rebrightening, though it would require a slow rise and a very rapid fall if they were correlated.

The rebrightening observed in the optical/NIR is about two orders of magnitude below the faint type Ic supernova ( $\mathrm{SN}$ ), 1994I and cannot be fit with standard SN templates because it requires a much earlier rise-time and a quicker decay than observed in SNe. If the flares in the optical and X-ray are associated, this probably also excludes a SN origin for the rebrightening, since the X-ray flare is so late. 
The very late time of these flares seems to exclude models related to the natural timescale of a compact-body merger (Rosswog et al. 2003; Setiawan et al. 2004; Oechslin \& Janka 2006), as well as those involving shock heating of a stellar companion unless the orbital distance is much larger than suggested by MacFadyen et al. (2005). Models where the characteristic spectrum is thermal cannot explain both the X-ray and optical flares together. The late time of the flaring may also be problematic for models involving large non-uniformity in the accretion (Perna et al. 2006; King et al. 2005), since the accretion must continue for $>10$ days after the burst.

\subsection{Jet breaks in SGRBs lightcurves}

The steep decay reported here could be indicative of a jet-break prior to the start of optical observations in GRB 050709, however, the X-ray data are well-fit (reduced $\chi^{2}=0.7$ ) by a single power-law decay from the HETE-WXM detection of the long soft emission $100 \mathrm{~s}$ after the short burst (Villasenor et al. 2005), to the late Chandra detection at 16.1 days (excluding the flare at 16.0 days $)$, with a decay index $\left(\alpha_{X}=1.97 \pm 0.02\right)$ which is close to the optical decay. This indicates that a break at early times ( $<2$ days) is unlikely. In this case, we can limit any achromatic break to $>10$ days. Indeed, it seems likely that there was no break as late as the third HST detection at 18.6 days, since the detection at this time, and in the X-ray at 16 days, are consistent with the single early power-laws. However we cannot absolutely exclude that such a break occurred around the time of the flaring, with the flare disguising such a break. Therefore the conservative limit on any break is $>10$ days. This limit corresponds to a half opening angle, $\theta_{\text {jet }}>23^{\circ}$, using the relation of Sari et al. (1999), an isotropic equivalent energy $E_{\text {iso }}=7 \times 10^{49} \mathrm{erg}$ (Fox et al. 2005) and assuming a density $n=10^{-2} \mathrm{~cm}^{-3}$. The limit is not very sensitive to the assumed density or the derived total energy since the angle is proportional to $\left(n / E_{\text {iso }}\right)^{0.125}$. The location of GRB 050709 in a star-forming galaxy suggests that the density is unlikely to be signicantly lower than assumed above, a higher density would result in a (slightly) larger limit on the opening angle. This limit, $\theta_{\text {jet }}>23^{\circ}$, is much larger than the typical opening angle found for LGRBs (Zeh et al. 2006).

While the lightcurves of SGRBs do decay rapidly, roughly as a power-law, they are all affected by strong variations, ranging from a moderate amplitude "wiggling" to very large amplitude flaring (e.g. GRB 050709, as noted above, or GRB 050724, Grupe et al. 2006). For this reason it is difficult to ascertain the decay slope of any underlying power-law and then fix an achromatic breaktime. This is evidenced by the first inaccurate suggestions of jet breaks in GRB 050709 and GRB 050724 (see Fig. 7 in Soderberg et al. 2006) - Grupe et al. (2006) report no lightcurve break detected in GRB 050724 either, out to at least three weeks after the burst. The break in the X-ray lightcurve of the afterglow of GRB 051221A at $\sim 5$ days may be a jet break (Burrows et al. 2006). This seems to be consistent with the available data (Soderberg et al. 2006), however without strong limits or detections at other wavelengths to indicate a simultaneous break, the claim that it is a jet break must be considered weak. The opening angle of $\sim 7^{\circ}$ (Burrows et al. 2006; Soderberg et al. 2006) corresponding to a jet break at 5 days must therefore be considered a lower limit. Evidence of an achromatic break in the lightcurve, critical to the analysis of the collimation of the outflows of SGRBs, has therefore yet to be observed in any SGRB.

Lower limits to achromatic break times are now available for two SGRBs with redshifts (GRB 050724 and GRB 051221A)

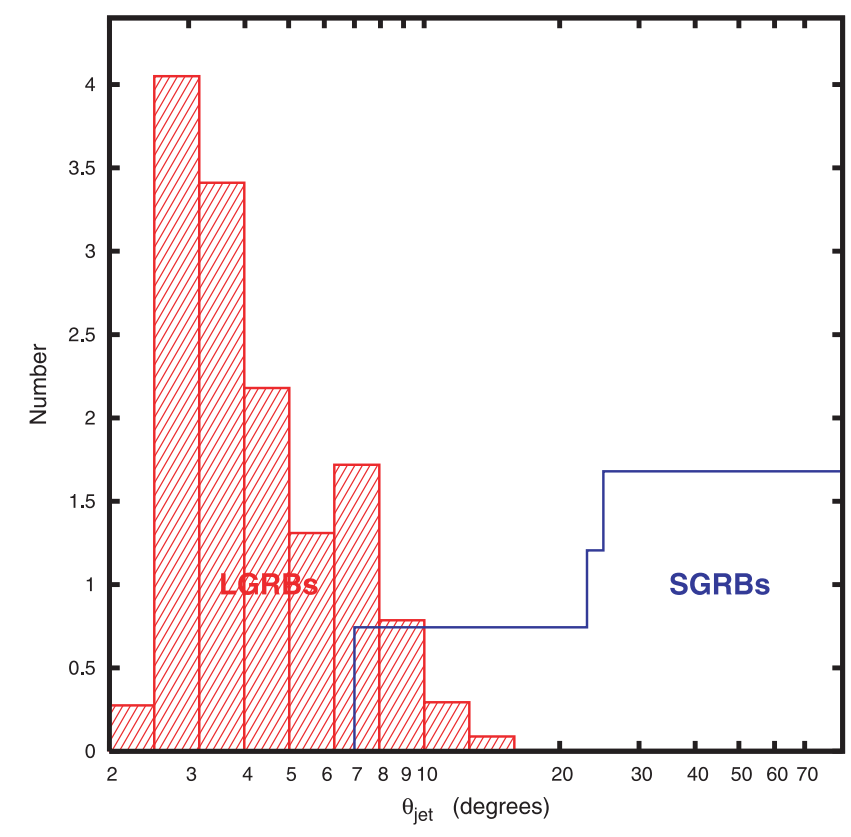

Fig. 2. Distributions of opening angles for short and long GRBs. Only lower limits are available for the short GRBs, but even with only four bursts, they seem clearly incompatible with the long GRB distribution. The long burst distribution is from the sample of Zeh et al. (2006). The short bursts are GRB $050709\left(>23^{\circ}\right.$, this paper), GRB $050724\left(>25^{\circ}\right.$, Grupe et al. 2006), GRB 051221A ( $>7^{\circ}$, Soderberg et al. 2006) and GRB $060121\left(>7^{\circ}\right.$ assuming $z=3$, Levan et al. 2006). The distributions have been produced by dividing the probability density for each opening angle between the bins using the available uncertainties and limits.

and one without (GRB 060121) ${ }^{1}$. Combining these limits with the limit for GRB 050709, we can compare their opening angles with the distribution of opening angles found for LGRBs (Fig. 2). It is immediately apparent that the distributions are different, with SGRBs having much larger opening angles, consistent with no collimation at all. While the opening angles are fairly insensitive to the assumed density, it might be possible to decrease the lower limits on the opening angle by as much as a factor of two if the assumed density could be lowered by a factor of about 300 . However, such low densities could be problematic in trying to reproduce the properties of the afterglows (e.g. Soderberg et al. 2006), and in the cases where the GRBs are found within a galaxy, such low densities can essentially be excluded.

The minimum $\gamma$-ray energies of the three SGRBs with known redshift is $1.6 \times 10^{49} \mathrm{erg}, 2.8 \times 10^{49} \mathrm{erg}$ and $1 \times 10^{49} \mathrm{erg}$ for GRB 050709, GRB 050724 and GRB 051221 A respectively. Their respective isotropic equivalent energies are $2 \times 10^{50} \mathrm{erg}$, $3 \times 10^{50}$ erg and $2.4 \times 10^{51}$ erg (Fox et al. 2005; Barthelmy et al. 2005; Soderberg et al. 2006). These limits are clearly different from the values found for classical LGRBs $\left(10^{50}-10^{52} \mathrm{erg}\right.$, Zeh et al. 2006). However, the SGRBs are substantially closer than most of the LGRBs in this sample. A comparison with the $\gamma$-ray energies of low-redshift LGRBs - e.g. GRB 980425 (Galama et al. 1998), 020903 (Sakamoto et al. 2004), 030329 (Hjorth et al. 2003), 031203 (Watson et al. 2006), 060218

1 The limits obtained for GRB 051210, GRB 050813 and GRB 050509B are very weak: none have an optical afterglow detection and therefore their redshifts are somewhat uncertain, and in all cases, X-ray emission is well-detected only in the first few hundred seconds after the burst. 
(Campana et al. 2006) - shows that the three limits for SGRBs substantially overlap with low-redshift LGRBs.

Models of short GRBs from neutron star (NS) mergers (Rosswog et al. 2003) seem naturally to produce wide opening angles for the neutrino-annihiliation driven outflow unless the baryonic wind from the remnant exerts significant confinement (Rosswog \& Ramirez-Ruiz 2003; Aloy et al. 2005). However, such wide opening angles could be problematic for the total energy released in such a scenario unless the efficiency is fairly high. Magnetic mechanisms may therefore be a more likely candidate to provide the energy release in NS-NS mergers (Price \& Rosswog 2006; Lee et al. 2005).

It is interesting to note that GRB $000301 \mathrm{C}$, suggested to be a SGRB (duration $2 \mathrm{~s}$ with a hard spectrum, Jensen et al. 2001), has an opening angle at the extreme end of the distribution for LGRBs (12 $\pm 1^{\circ}$, Zeh et al. 2006), as well as a strong (1 mag) deviation from a power-law decay at 4-5 days after the trigger. But at the same time, GRB $000301 \mathrm{C}$ is at a fairly high redshift, $z=2.04$, much further away than the known SGRB redshifts. At a much lower redshift, the duration of this burst would lie well within the SGRB range. GRB 000301C also has a damped Ly $\alpha$ (DLA) absorption system and extinction detected in its afterglow spectrum (Jensen et al. 2001), suggesting an actively star-forming galaxy. Two other bursts are worth noting in this discussion: GRB 001025A, an IPN-localised hard burst with duration 2.9s (Pedersen et al. 2006), and GRB 060206, also a hard burst with duration $7 \mathrm{~s}$ (Palmer et al. 2006). In the case of GRB 001025A it had a fast decay and no detected optical afterglow to a limit of $R>25.5$ at 1.2 days (Watson et al. 2002; Pedersen et al. 2006). For GRB 060206, the redshift is high ( $z=4.05$ Fynbo et al. 2006) - at low redshift, this GRB would have had a duration about as short as GRB 050724. It also shows huge variability in the optical (Stanek et al. 2006; Monfardini et al. 2006; Woźniak et al. 2006). Like GRB 000301C, its spectrum also has a DLA absorption system (Fynbo et al. 2006).

The strong variations observed in almost all short GRBs where there is even a moderate coverage of the lightcurve, make it difficult to determine breaks in the power-law decays. Indeed, there is a possibility that some lightcurves may be dominated by flaring, with little of the flux contributed by an underlying power-law decay. In cases with long-duration, large amplitude flaring, jet-break times would not be determined, leading to very large opening angle limits. However this explanation of large opening angles seems unlikely in most cases and is contradicted by the detection of a clean, relatively slow power-law decay in the optical in GRB 050709 and in the X-ray in GRB 051221A.

\section{Conclusions}

The SGRB 050709 was the first GRB with a detected optical afterglow (Hjorth et al. 2005b). It is the only SGRB where clear evidence for a jet break in the optical lightcurve has been claimed. We have shown that the optical decay of this GRB follows a single steep power-law decay with a rebrightening at $\sim 10$ days. There is no evidence of a jet break. The optical rebrightening in GRB 050709 is not easily compatible with models involving supernovae, shock heating of a stellar companion or non-uniformities in the accretion disk. So far there is no compelling evidence for a jet break in any SGRB and available limits are not compatible with the distribution of opening angles in long GRBs. There is no strong evidence for collimation in short bursts, implying that short GRBs may be more energetic than previously believed.
Acknowledgements. We are grateful to Andreas Zeh for providing the opening angle determinations for long GRBs. The Dark Cosmology Centre is funded by the DNRF. We acknowledge benefits from collaboration within the EU FP5 Research Training Network, "Gamma-Ray Bursts: An Enigma and a Tool".

\section{References}

Aloy, M. A., Janka, H.-T., \& Müller, E. 2005, A\&A, 436, 273

Barthelmy, S. D., Chincarini, G., Burrows, D. N., et al. 2005, Nature, 438, 994

Berger, E., Price, P. A., Cenko, S. B., et al. 2005, Nature, 438, 988

Burrows, D. N., Grupe, D., Capalbi, M., et al. 2006 [arXiv:astro-ph/0604320]

Campana, S., Mangano, V., Blustin, A. J., et al. 2006 [arXiv: astro-ph/0603279]

Castro-Tirado, A. J., de Ugarte Postigo, A., Gorosabel, J., et al. 2005, A\&A, 439, L15

Cobb, B. E., Bailyn, C. D., van Dokkum, P. G., \& Natarajan, P. 2006 [arXiv: astro-ph/0603832]

Covino, S., Malesani, D., Israel, G. L., et al. 2006, A\&A, 447, L5

Dezalay, J.-P., Barat, C., Talon, R., et al. 1992, in Amer. Inst. Phys. Conf. Ser., 304

Fox, D. B., Frail, D. A., Price, P. A., et al. 2005, Nature, 437, 845

Fynbo, J. P. U., Starling, R. L. C., Ledoux, C., et al. 2006, A\&A, 451, L47 Galama, T. J., Vreeswijk, P. M., van Paradijs, J., et al. 1998, Nature, 395, 670

Gehrels, N., Sarazin, C. L., O'Brien, P. T., et al. 2005, Nature, 437, 851

Gorosabel, J., Castro-Tirado, A. J., Guziy, S., et al. 2006, A\&A, 450, 87

Grupe, D., Burrows, D. N., Patel, S. K., et al. 2006

[arXiv: astro-ph/0603773]

Hjorth, J., Sollerman, J., Gorosabel, J., et al. 2005a, ApJ, 630, L117

Hjorth, J., Sollerman, J., Møller, P., et al. 2003, Nature, 423, 847

Hjorth, J., Watson, D., Fynbo, J. P. U., et al. 2005b, Nature, 437, 859

Jakobsson, P., Hjorth, J., Fynbo, J. P. U., et al. 2004, A\&A, 427, 785

Jensen, B. L., Fynbo, J. U., Gorosabel, J., et al. 2001, A\&A, 370, 909

King, A., O’Brien, P. T., Goad, M. R., et al. 2005, ApJ, 630, L113

Kouveliotou, C., Meegan, C. A., Fishman, G. J., et al. 1993, ApJ, 413, L101

La Parola, V., Mangano, V., Fox, D., et al. 2006, [arXiv:astro-ph/0602541]

Lazzati, D., Ramirez-Ruiz, E., \& Ghisellini, G. 2001, A\&A, 379, L39

Lee, W. H., Ramirez-Ruiz, E., \& Granot, J. 2005, ApJ, 630, L165

Levan, A. J., Tanvir, N. R., Fruchter, A. S., et al. 2006

[arXiv: astro-ph/0603282]

MacFadyen, A. I., Ramirez-Ruiz, E., \& Zhang, W. 2005

[arXiv: astro-ph/0510192]

Malesani, D., Tagliaferri, G., Chincarini, G., et al. 2004, ApJ, 609, L5

Mirabal, N., Halpern, J. P., An, D., Thorstensen, J. R., \& Terndrup, D. M. 2006, ApJ, 643, L99

Modjaz, M., Stanek, K. Z., Garnavich, P. M., et al. 2006 [arXiv: astro-ph/0603377]

Monfardini, A., Kobayashi, S., Guidorzi, C., et al. 2006 [arXiv:astro-ph/0603181]

Norris, J. P., Cline, T. L., Desai, U. D., \& Teegarden, B. J. 1984, Nature, 308, 434

Oechslin, R., \& Janka, H.-T. 2006, MNRAS, 368, 1489

Palmer, D., Barbier, L., Barthelmy, S., et al. 2006, GCN Circ., 4697

Panaitescu, A. 2006, MNRAS, 367, L42

Panaitescu, A., Kumar, P., \& Narayan, R. 2001, ApJ, 561, L171

Pedersen, K., Hurley, K., Hjorth, J., et al. 2006, ApJ, 636, 381

Perna, R., Armitage, P. J., \& Zhang, B. 2006, ApJ, 636, L29

Pian, E., Mazzali, P. A., Masetti, N., et al. 2006, [arXiv:astro-ph/0603530]

Price, D. J., \& Rosswog, S. 2006, Science, 312, 719

Prochaska, J. X., Bloom, J. S., Chen, H.-W., et al. 2006, ApJ, 642, 989

Rosswog, S., \& Ramirez-Ruiz, E. 2003, MNRAS, 343, L36

Rosswog, S., Ramirez-Ruiz, E., \& Davies, M. B. 2003, MNRAS, 345, 1077

Sakamoto, T., Lamb, D. Q., Graziani, C., et al. 2004, ApJ, 602, 875

Sari, R., Piran, T., \& Halpern, J. P. 1999, ApJ, 519, L17

Sari, R., Piran, T., \& Narayan, R. 1998, ApJ, 497, L17

Setiawan, S., Ruffert, M., \& Janka, H.-T. 2004, MNRAS, 352, 753

Soderberg, A. M., Berger, E., Kasliwal, M., et al. 2006

[arXiv: astro-ph/0601455]

Sollerman, J., Jaunsen, A. O., Fynbo, J. P. U., et al. 2006

[arXiv: astro-ph/0603495]

Stanek, K. Z., Dai, X., Prieto, J. L., et al. 2006, [arXiv:astro-ph/0602495]

Stanek, K. Z., Matheson, T., Garnavich, P. M., et al. 2003, ApJ, 591, L17

Villasenor, J. S., Lamb, D. Q., Ricker, G. R., et al. 2005, Nature, 437, 855

Watson, D., Reeves, J. N., Osborne, J., et al. 2002, A\&A, 393, L1

Watson, D., Vaughan, S. A., Willingale, R., et al. 2006, ApJ, 636, 967

Willingale, R., Osborne, J. P., O’Brien, P. T., et al. 2004, MNRAS, 349, 31

Woźniak, P. R., Vestrand, W. T., Wren, J. A., et al. 2006, ApJ, 642, L99

Zeh, A., Klose, S., \& Kann, D. A. 2006, ApJ, 637, 889 